HUTP-99/A025, NUB 3201

\title{
A Remark on Brane Stabilization in Brane World
}

\author{
Zurab Kakushadze* \\ Jefferson Laboratory of Physics, Harvard University, Cambridge, MA 02138 \\ and \\ Department of Physics, Northeastern University, Boston, MA 02115
}

(June 28, 1999)

\begin{abstract}
In this note we discuss dynamical mechanisms for brane stabilization in the brane world context. In particular, we consider supersymmetry preserving brane stabilization, and also brane stabilization accompanied by supersymmetry breaking. These mechanisms are realized in some four dimensional $\mathcal{N}=1$ supersymmetric orientifold models. For illustrative purposes we consider two explicit orientifold models previously constructed in [1]. In both of these models branes are stabilized at a finite distance from the orientifold planes. In the first model brane stabilization occurs via supersymmetry preserving non-perturbative gauge dynamics. In the second model supersymmetry is dynamically broken, and brane stabilization is due to an interplay between non-perturbatively generated superpotential and tree-level Kähler potential.
\end{abstract}

*E-mail: zurab@string.harvard.edu. Address after September 1, 1999: C.N. Yang Institute for Theoretical Physics, State University of New York, Stony Brook, NY 11794. 
The discovery of D-branes [2] is likely to have important phenomenological implications. Thus, the Standard Model gauge and matter fields may live inside of $p \leq 9$ spatial dimensional $p$-branes, while gravity lives in a larger (10 or 11) dimensional bulk of the space-time. This "Brane World" picture] a priori appears to be a viable scenario, and, based on considerations of gauge and gravitational coupling unification, dilaton stabilization and weakness of the Standard Model gauge couplings, in [9] it was actually argued to be a likely description of nature. In particular, these phenomenological constraints seem to be embeddable in the brane world scenario (with the Standard Model fields living on branes with $3<p<9$ ), which therefore might provide a coherent picture for describing our universe [9]. This is largely due to a much higher degree of flexibility of the brane world scenario compared with, say, the old perturbative heterotic framework.

An important question arising in the brane world context is the issue of brane stabilization. In particular, in many four dimensional $\mathcal{N}=1$ supersymmetric Type I/Type I' vacua there are flat directions corresponding to the positions of branes either with respect to each other or to the orientifold planes. Perturbatively, therefore, branes are not stabilized in such vacua. Upon supersymmetry breaking we might generically expect brane stabilization to occur. However, brane stabilization can sometimes occur via supersymmetry preserving nonperturbative dynamics. The purpose of this note is to illustrate some possible mechanisms of brane stabilization in the brane world context. In particular, brane stabilization in the mechanisms we discuss here is due to non-perturbative gauge dynamics in the world-volume of the branes, and can be supersymmetry preserving as well as supersymmetry breaking dynamics. In the latter case the VEV of the field whose F-term breaks supersymmetry is precisely the inter-brane distance or the distance between the branes and an orientifold plane. For illustrative purposes we present explicit string models where these mechanisms are realized. These examples, which are consistent string vacua (with four non-compact and six compact space-time dimensions) satisfying the requirements of tadpole and anomaly cancellation, were originally constructed in [1], and here we briefly review these constructions. In particular, the models we consider in this paper are four dimensional $\mathcal{N}=1$ supersymmetric orientifolds with non-trivial NS-NS $B$-flux.

Here we should emphasize that in the following discussions we will assume that dilaton is somehow stabilized via some other dynamics whose relevant scale is higher than the strong scale of the gauge dynamics responsible for brane stabilization. We can then safely integrate dilaton out and consider brane stabilization for a fixed gauge coupling determined by the dilaton VEV. On the other hand, if dilaton stabilization is not achieved, one runs into the usual runaway problem - the ground state of the theory is located at infinitely weak coupling where branes are destabilized and supersymmetry is restored (in the cases where brane stabilization is accompanied by supersymmetry breaking). The examples we discuss in this note, therefore, should only be understood as illustrative (toy) representatives of $\mathcal{N}=1$ gauge theories (arising from consistent string vacua) where non-perturbative dynamics can stabilize the VEV of a field which in the brane world context is interpreted as measuring

\footnotetext{
${ }^{1}$ For recent developments, see, e.g., [3 9]. The brane world picture in the effective field theory context was discussed in [10]. TeV-scale compactifications were originally discussed in [11] in the context of supersymmetry breaking.
} 
the separation between branes or branes and orientifold planes.

Let us begin our discussion with the case where brane stabilization is accompanied by supersymmetry breaking. Thus, consider an $\mathcal{N}=1$ supersymmetric gauge theory with $S U(4)$ gauge group and matter consisting of one chiral superfield $\Phi$ transforming in the two-index antisymmetric irrep 6 of $S U(4)$. Note that this theory is anomaly free. There is a flat direction in this theory along which $\Phi$ acquires a VEV as follows: $\langle\Phi\rangle=\operatorname{diag}(X \epsilon, X \epsilon)$, where $\epsilon \equiv i \sigma_{2}$ is a $2 \times 2$ antisymmetric matrix ( $\sigma_{2}$ is a Pauli matrix). The gauge group along this flat direction is broken down to $S p(4) \approx S O(5)$, and the only matter left is a singlet of $S p(4)$ (which is precisely the chiral superfield acquiring the $\mathrm{VEV} X$ ). Thus, below the mass scale $X$ we have pure $\mathcal{N}=1$ supersymmetric gauge theory with the gauge group $S p(4)$ plus the singlet.

Supersymmetry in this model is actually broken. In fact, this is already the case in the context of global supersymmetry. The reason for this is that the gaugino condensate in the $S p(4)$ gauge theory depends non-trivially on $X$. This can be seen as follows. The gauge coupling running starts at the string scale $M_{s}$ (which we will identify as the UV cut-off of the theory). Between the scales $M_{s}$ and $X$ (where we assume $X \ll M_{s}$ ) the gauge coupling of the $S U(4)$ gauge theory runs with the one-loop $\beta$-function coefficient $b_{0}=11$. Below the mass scale $X$ the gauge coupling of the $S p(4)$ gauge theory runs with the one-loop $\beta$-function coefficient $\widetilde{b}_{0}=9$. Matching the two gauge couplings at the scale $X$ then gives the following strong scale $\widetilde{\Lambda}$ of the $S p(4)$ theory:

$$
\widetilde{\Lambda}=\Lambda^{b_{0} / \widetilde{b}_{0}} X^{1-b_{0} / \widetilde{b}_{0}}=\Lambda^{11 / 9} X^{-2 / 9}
$$

where $\Lambda \equiv M_{s} \exp \left(-2 \pi S / b_{0}\right)$ is the strong scale of the original $S U(4)$ gauge theory. (Here $S \equiv 4 \pi / g^{2}+i \theta / 2 \pi$ is the dilaton $\mathrm{VEV}$ which determines the $S U(4)$ gauge coupling $g$ at the string scale as well as the vacuum angle $\theta$.) The gaugino condensation in the $S p(4)$ theory generates the following superpotentiall]

$$
W=3 \widetilde{\Lambda}^{3}=3 \Lambda^{11 / 3} X^{-2 / 3} .
$$

Note that this superpotential has runaway behavior in $X$ so that for any finite $X$ supersymmetry is broken. In the context of global supersymmetry the vacuum is located at infinite $X$ where supersymmetry is restored. In the locally supersymmetric context, however, the situation is quite different.

The key point here is the following. Thus, as was pointed out in [12], once such a theory with a runaway direction is coupled to supergravity, generically there is a natural shut-down scale for runaway directions, namely, the Planck scale. More precisely, this is the case for a certain large class of Kähler potentials, which, in particular, includes Kähler potentials of

\footnotetext{
${ }^{2}$ Note that for a pure $\mathcal{N}=1$ supersymmetric gauge theory with a simple gauge group $G$ the non-perturbative superpotential generated by the gaugino condensation (in the $\overline{\mathrm{DR}}$ subtraction scheme) is given by $W=h_{G} \Lambda_{G}^{3}$, where $h_{G}$ is the dual Coxeter number of the group $G$ ( $h_{G}=N$ for $G=S U(N), h_{G}=N-2$ for $G=S O(N), h_{G}=N+1$ for $G=S p(2 N)$, and in our conventions $S p(2 N)$ has rank $N)$, and $\Lambda_{G} \equiv \exp \left(-2 \pi S / b_{0}(G)\right)$ is the strong scale of the theory (here $\left.b_{0}(G)=3 h_{G}\right)$.
} 
minimal type. Local supersymmetry breaking and stabilization of the runaway directions is then due to the interplay between non-perturbatively generated runaway superpotential and tree-level Kähler potential.

Let us illustrate the above point by considering a theory in which there is a runaway superpotential $W=c / X^{\alpha}$ generated via some non-perturbative dynamics. Let us assume that the Kähler potential for $X$ is minimal: $K=X X^{*}$. For simplicity in the following we will adapt the units where the reduced Planck scale $M_{P}=1 / \sqrt{8 \pi G_{N}}$ is set to one, so that the scalar potential reads:

$$
V=e^{K}\left(K_{X X^{*}}^{-1}\left|F_{X}\right|^{2}-3|W|^{2}\right)
$$

where the F-term $F_{X}=W_{X}+K_{X} W$. Let $\rho \equiv X X^{*}$. Then we have

$$
V(\rho)=|c|^{2} e^{\rho}\left(\frac{\alpha^{2}}{\rho^{\alpha+1}}-\frac{2 \alpha+3}{\rho^{\alpha}}+\frac{1}{\rho^{\alpha-1}}\right) .
$$

The extrema of this scalar potential are located at $\rho_{0}=\alpha$ and $\rho_{ \pm}=\alpha+1 \pm \sqrt{\alpha+1}$. Here we are assuming that $\alpha>0$. Then the extremum at $\rho=\rho_{0}$ corresponds to a supersymmetric maximum with vanishing F-term. The extrema at $\rho=\rho_{ \pm}$correspond to minima with broken supersymmetry. Thus, in such a theory $X$ is indeed stabilized and supersymmetry is broken. Note that in the runaway superpotential in (2) generated in the $S U(4)$ gauge theory with one chiral 6 which we discussed above we have $\alpha=2 / 3$.

Note, however, that the superpotential (2) is only valid for $X \ll M_{s}$, whereas the above minima correspond to the values of $X \sim M_{P}$ (here we can assume for simplicity that the reduced Planck scale and the string scale are of the same order of magnitude). So, strictly speaking, the above quantitative analysis is not valid. However, the qualitative conclusion that $X$ is stabilized and supersymmetry is broken is still correct. The reason for this is the following. Note that for $X \gg M_{s}$ we would have pure $S p(4)$ super-Yang-Mills theory below the cut-off scale $M_{s}$. Then the superpotential generated in this case would be independent of $X: W=3 \widetilde{\Lambda}^{3}$, where $\widetilde{\Lambda} \equiv M_{s} \exp \left(-2 \pi S / \widetilde{b}_{0}\right)$. The corresponding scalar potential (in the assumption of the minimal Kähler potential) is then given by

$$
V(\rho)=|W|^{2} e^{\rho}(\rho-3) .
$$

This scalar potential can be seen to stabilize $\rho$ at $\rho=2$, where the F-term is non-zero, hence supersymmetry breaking. For intermediate values of $X$ (that is, $X \sim M_{s}$ ), the superpotential is a non-trivial function of $X$ interpolating between $\propto X^{-2 / 3}$ for small $X$ and a constant for large $X$. (This interpolating function depends on the details of various threshold corrections around the scale $M_{s}$.) From the limiting behavior, however, it is clear

\footnotetext{
${ }^{3}$ Here we should point out that for $X \gg M_{s}$ we must also take into account the fact that generically dilaton may not be constant, but vary logarithmically in the two transverse directions corresponding to the motion of the branes. This would modify the $X$ dependence in $V$, but it is not difficult to see that the qualitative conclusions on brane stabilization at $X \sim M_{s}$ would still remain correct.
} 
that $X$ is stabilized and supersymmetry is broken (subject to the appropriate assumptions on the Kähler potential). As we have already mentioned, in the following we will present an explicit string model whose low energy effective field theory contains the $S U(4)$ gauge theory with one chiral 6 discussed above, and where the $\operatorname{VEV} X$ is identified with the separation between D-branes and the corresponding orientifold plane.

Next, we would like to discuss an example where brane stabilization occurs via supersymmetry preserving dynamics. Thus, consider an $\mathcal{N}=1$ supersymmetric gauge theory with $S U(4) \otimes S U(4)$ gauge group and matter consisting of one chiral superfield $\Phi$ transforming in $(\mathbf{1 0}, \mathbf{1})$ of $S U(4) \otimes S U(4)$, one chiral superfield $Q$ transforming in $(\overline{4}, \mathbf{4})$, and one chiral superfield $\widetilde{Q}$ transforming in $(\overline{\mathbf{4}}, \overline{\mathbf{4}})$. Note that this theory is anomaly free. We will assume that there is a tree-level superpotential given by

$$
W_{\text {tree }}=\lambda \Phi Q \widetilde{Q},
$$

where $\lambda$ is the corresponding Yukawa coupling. There are three flat directions in this model. One corresponds to the field $Q$ acquiring a non-zero VEV. The gauge group along the flat direction $\langle Q\rangle \neq 0$ is broken down to $S U(4)_{\text {diag }} \subset S U(4) \otimes S U(4)$. After the gauge symmetry breaking we have a singlet of $S U(4)_{\text {diag }}$ coming from $Q$, and also one chiral 6 of $S U(4)_{\text {diag }}$ coming from $\widetilde{Q}$. The rest of the fields in $Q$ are eaten in the super-Higgs mechanism, whereas the rest of the fields in $\widetilde{Q}$ pair up with those in $\Phi$ and become heavy via the corresponding coupling in the tree-level superpotential (6). Thus, along this flat direction we have the $S U(4)$ gauge theory with one chiral 6 plus a singlet. This is the same theory as that already discussed above except for the presence of the extra singlet. One can analyze this case in a similar fashion, however, and it is not difficult to see that VEV stabilization is accompanied by supersymmetry breaking just as in the previous case. Note that the second flat direction along which $\langle\widetilde{Q}\rangle \neq 0$ gives rise to the same theory as above.

In the following we will therefore be interested in the third flat direction along which $\langle\Phi\rangle=\operatorname{diag}(X, X, X, X)$ and the gauge group is broken down to $S O(4) \otimes S U(4)$. The only matter left is a singlet (coming from $\Phi$ ) which is precisely the chiral superfield acquiring the VEV $X$. The rest of the fields in $\Phi$ are eaten in the super-Higgs mechanism, and the fields $Q, \widetilde{Q}$ acquire mass $\lambda X$ due to the corresponding coupling in the tree-level superpotential (6). Thus, at low energies we have gaugino condensates in both $S O(4) \approx S U(2) \otimes S U(2)$ and $S U(4)$ subgroups. Matching the gauge couplings of $[S U(2) \otimes S U(2)] \otimes S U(4)$ with the original gauge couplings of $S U(4) \otimes S U(4)$ at the corresponding threshold scales, we obtain the following non-perturbative superpotential on this branch of the classical moduli space (here we assume that gauge couplings of both $S U(4)$ subgroups are the same at the string scale):

$$
W=2 \lambda^{4} X^{-2} \Lambda^{5}+4 \lambda X\left(\Lambda^{\prime}\right)^{2},
$$

where $\Lambda \equiv M_{s} \exp \left(-2 \pi S / b_{0}\right)$, and $\Lambda^{\prime} \equiv M_{s} \exp \left(-2 \pi S / b_{0}^{\prime}\right)$. Here $b_{0}=5$ and $b_{0}^{\prime}=8$ are the one-loop $\beta$-function coefficients of the first respectively second $S U(4)$ subgroups above the corresponding threshold scales.

In fact, we can generalize the above model as follows. Thus, consider an $\mathcal{N}=1$ supersymmetric gauge theory with $S U(M) \otimes S U(N)$ gauge group and matter consisting of one chiral superfield $\Phi$ transforming in $\left(\mathbf{R}_{\eta}, \mathbf{1}\right)$ of $S U(M) \otimes S U(N)$, one chiral superfield $Q$ transforming in $(\overline{\mathbf{M}}, \mathbf{N})$, and one chiral superfield $\widetilde{Q}$ transforming in $(\overline{\mathbf{M}}, \overline{\mathbf{N}})$. Here $\mathbf{R}_{\eta}=\mathbf{S}$ 
for $\eta=+1$, and $\mathbf{R}_{\eta}=\mathbf{A}$ for $\eta=-1$, where $\mathbf{S}$ is the two-index symmetric $(M(M+1) / 2$ dimensional) representation of $S U(M)$, while $\mathbf{A}$ is the two-index antisymmetric $(M(M-1) / 2$ dimensional) representation of $S U(M)$. Anomaly cancellation requires that $M=2 N-4 \eta$. We will assume that there is a tree-level superpotential given by (6). Along the flat direction $\langle\Phi\rangle \neq 0$ the gauge group is broken down to $G_{\eta}(M) \otimes S U(N)$, where $G_{\eta}(M)=S O(M)$ for $\eta=+1$, and $G_{\eta}(M)=S p(M)$ for $\eta=-1$. The only matter left is a singlet coming from $\Phi$. The gaugino condensation in both $G_{\eta}(M)$ and $S U(N)$ subgroups gives rise to the following non-perturbative superpotential:

$$
W=C(N, \eta) \lambda^{\frac{N}{N-3 \eta}} X^{-\frac{N-2 \eta}{N-3 \eta}} \Lambda^{\frac{4 N-11 \eta}{N-3 \eta}}+N(\lambda X)^{2-\frac{4 \eta}{N}}\left(\Lambda^{\prime}\right)^{1+\frac{4 \eta}{N}}
$$

where $\Lambda^{4 N-11 \eta} \equiv M_{s} \exp (-2 \pi S)$, and $\left(\Lambda^{\prime}\right)^{N+4 \eta} \equiv M_{s} \exp (-2 \pi S)$ (and we are assuming that gauge couplings of both $S U(M)$ and $S U(N)$ are the same at the string scale). Here $C(N, \eta)=N-3 \eta$ except when $N=4, \eta=+1$. In the latter case $G_{\eta}(M)=S O(4) \approx S U(2) \otimes$ $S U(2)$ (that is, $G_{\eta}(M)$ is not simple), and $C(N, \eta)=2$ (instead of 1 ) as we have to add the contributions of gaugino condensates in both $S U(2)$ subgroups. (Here we are assuming that the phases of the gaugino condensates are the same for both $S U(2)$ subgroups.) We note that in deriving the superpotential (8) some care is needed. In particular, for $\eta=-1$ we have the breaking $S U(M) \supset S p(M)$, and the $S p(M)$ gauge coupling at the scale $X$ is the same as that of $S U(M)$. However, for $\eta=+1$ we have the breaking $S U(M) \supset S O(M)$, and the $S O(M)$ gauge coupling $\widetilde{g}(X)$ at the scale $X$ is related to the $S U(M)$ gauge coupling $g(X)$ at the same scale via $2 \widetilde{g}^{2}(X)=g^{2}(X)$. This is due to the special embedding of $S O(M)$ into $S U(M)$. (Thus, in the perturbative heterotic language $S O(M)$ in this case is realized via a level-2 current algebra.) In particular, this is important in obtaining the correct powers of $X$ and $\Lambda$ as well as the numerical coefficient $C(N, \eta)$ in the first term in (8).

Let us go back to the non-perturbative superpotential (7) in the $S U(4) \otimes S U(4)$ example discussed above. Let us first consider the globally supersymmetric setup. The F-term of $X$ vanishes for

$$
X=\lambda\left(\frac{\Lambda^{5}}{\left(\Lambda^{\prime}\right)^{2}}\right)^{1 / 3}=\lambda M_{s} \exp (-\pi S / 2)
$$

For a large enough dilaton VEV $S$ (that is, for small enough gauge coupling at the string scalef) we have $X \ll M_{s}$, so that the above quantitative analysis is valid in the globally supersymmetric context. That is, the $\mathrm{VEV} X$ is stabilized via supersymmetry preserving dynamics. Here we would like to address the issue of whether the same is true once we couple this system to supergravity.

To answer the above question, consider a generic system of superfields $\Phi_{i}$ with the superpotential $W$. Let $K(\Phi, \bar{\Phi})$ be the Kähler potential, which we will assume to be non-singular

\footnotetext{
${ }^{4}$ Note that large $S$ in our conventions does not necessarily imply weak string coupling. Thus, as was pointed out in [9], the string coupling can be of order 1, while the gauge coupling (at the string scale) of a gauge theory arising from a set of $p$-branes compactified on a $p-3$ dimensional space can be small if the corresponding compactification volume $V_{p-3}$ is large in the string units. This volume is absorbed in the above definition of the dilaton VEV $S$.
} 
for non-zero finite values of $\Phi_{i}$. The scalar potential is given by (once again, here we set the reduced Planck scale $M_{P}$ to one):

$$
V=e^{K}\left(G_{i \bar{j}} F_{i}\left(F_{j}\right)^{*}-3 W W^{*}\right)
$$

where the summation over repeated indices is understood, $G_{i \bar{j}} \equiv K_{i \bar{j}}^{-1}$ is the inverse of the Kähler metric, $F_{i} \equiv W_{i}+K_{i} W$ are the F-terms for the fields $\Phi_{i}$, and $W_{i} \equiv \partial W / \partial \Phi_{i}$.

Note that if all of the F-terms $F_{i}$ are vanishing, local supersymmetry is unbroken. It is easy to verify that a point with $F_{i} \equiv 0$ corresponds to an extremum of the scalar potential. (In this case $V_{i}=V_{\bar{i}}=0$.) We would like to investigate the conditions under which this extremum is actually a local minimum of the scalar potential. To do this we need the following quantities evaluated at the point $F_{i} \equiv 0$ :

$$
\begin{aligned}
& V_{i j}=-e^{K} F_{i j} W^{*}, \\
& V_{i \bar{j}}=e^{K}\left[G_{i^{\prime} \bar{j}^{\prime}} F_{i^{\prime} i}\left(F_{j^{\prime} j}\right)^{*}-2 K_{i \bar{j}} W W^{*}\right] .
\end{aligned}
$$

Here $F_{i j} \equiv\left(F_{i}\right)_{j}=W_{i j}+\left(K_{i j}-K_{i} K_{j}\right) W$. Note that if, say, the absolute values of all the eigenvalues of the matrix $W_{i j}$ are much larger than $|W|$ at the point $F_{i} \equiv 0$, then this point corresponds to a local minimum of the scalar potential?.

The above discussion has the following implications for the fate of globally supersymmetric minima corresponding to the superpotential $W$ in the local supergravity context. Suppose the F-flatness conditions $W_{i}=0$ in the global setting have a solution with all the VEVs stabilized at $\Phi_{i}=\Phi_{i}^{(0)}$. Furthermore, suppose that the eigenvalues of the matrix $W_{i j}\left(\Phi^{(0)}\right)$ are all non-zero, and their absolute values are much larger than $\left|W\left(\Phi^{(0)}\right)\right|$. Then in the vicinity of the point $\Phi_{i}^{(0)}$ there is another point $\Phi_{i}^{(1)}$ which is a locally supersymmetric minimum of the scalar potential (10). The proof of this statement is very simple. First, the F-flatness conditions in the local setting are given by $W_{i}(\Phi)=-K_{i}(\Phi, \bar{\Phi}) W(\Phi)$. Since $W_{i j}\left(\Phi^{(0)}\right)$ is non-singular, these equations have a solution at $\Phi_{i}=\Phi_{i}^{(1)}$, where $\Phi_{i}^{(1)}=\Phi_{i}^{(0)}-M_{i j}\left(\Phi^{(0)}\right) K_{j}\left(\Phi^{(0)}, \bar{\Phi}^{(0)}\right) W\left(\Phi^{(0)}\right)+\mathcal{O}\left(\epsilon^{2}\right)$, and $\epsilon<<1$ is the absolute value of the largest eigenvalue of the matrix $M_{i j}\left(\Phi^{(0)}\right) W\left(\Phi^{(0)}\right)$, where $M_{i j}\left(\Phi^{(0)}\right)$ is the matrix inverse to $W_{i j}\left(\Phi^{(0)}\right)$. Moreover, according to our discussion above, the point $\Phi_{i}^{(1)}$ corresponds to a local minimum. Here we note that the physical reason for this is clear - since in the globally supersymmetric setup all the fields are heavy at the minimum, there is no Goldstino candidate required for local supersymmetry breaking once we couple the system to supergravity.

Let us now go back to the superpotential (7), and see whether the aforementioned conditions are satisfied at the global minimum given by (9). Thus, we have

\footnotetext{
${ }^{5}$ One can easily write down the precise condition for the minimum which does not require the absolute values of the eigenvalues of the matrix $W_{i j}$ be much larger than $|W|$, but satisfy certain inequalities.

${ }^{6}$ Here we assume that the Kähler potential and its derivatives are of order 1 or smaller at $\Phi_{i}=\Phi_{i}^{(0)}$, which is usually the case.
} 


$$
\left|W_{X X} / W\right|=2|\lambda|^{-2} M_{s}^{-2} \exp (\pi \operatorname{Re}(S)) \gg 1
$$

Here we are using the $M_{P}=1$ units, and we are assuming that the Yukawa coupling is of order of the gauge coupling at the string scale: $|\lambda| \sim g$. Thus, in this model the $\mathrm{VEV} X$ is indeed stabilized without supersymmetry breaking, and the stabilized value of $X$ is much smaller than $M_{s}$ (for large enough values of $S$ ).

Next, as we promised in the beginning, we are going to give the explicit string construction of the above models. In fact, these models were already constructed in [1] where a more detailed discussion can be found. Here we will briefly review the construction in [1], and identify various flat directions in the classical moduli space with the motion of D-branes in the corresponding transverse directions.

Consider Type I compactified on the following orbifold Calabi-Yau three-fold with $S U(3)$ holonomy: $\mathcal{M}_{3} \equiv\left(T^{2} \otimes T^{2} \otimes T^{2}\right) /\left(\mathbf{Z}_{3} \otimes \mathbf{Z}_{3}\right)$. Let $g_{1}$ and $g_{2}$ be the generators of the two $\mathbf{Z}_{3}$ subgroups. Their action on the complex coordinates $z_{1}, z_{2}, z_{3}$ parametrizing the three two-tori is given by:

$$
\begin{aligned}
& g_{1} z_{1}=\omega z_{1}, \quad g_{1} z_{2}=\omega^{-1} z_{2}, \quad g_{1} z_{3}=z_{3}, \\
& g_{2} z_{1}=z_{1}, \quad g_{2} z_{2}=\omega z_{2}, \quad g_{2} z_{3}=\omega^{-1} z_{3} .
\end{aligned}
$$

Here $\omega \equiv \exp (2 \pi i / 3)$. This Calabi-Yau three-fold has the Hodge numbers $\left(h^{1,1}, h^{2,1}\right)=$ $(84,0)$, so there are 84 chiral supermultiplets in the closed string sector. In the open string sector we have D9-branes only whose number depends on the rank $b$ of the NS-NS $B$-field $B_{i j}(i, j=1, \ldots, 6$ label the real compact directions corresponding to complex coordinates $\left.z_{1}, z_{2}, z_{3}\right)$. Note that since $B_{i j}$ is antisymmetric, $b$ can only take even values $0,2,4,6$. The untwisted tadpole cancellation conditions then imply that we have $2^{5-b / 2}$ D9-branes 13 . In the following we are going to be interested in the cases $b=2$ and $b=4$.

- $b=2$. We have 16 D9-branes. The orientifold projection is of the Sp type. The solution to the twisted tadpole cancellation conditions (up to equivalent representations) is given by! [1]:

$$
\begin{aligned}
& \gamma_{g_{1}, 9}=\operatorname{diag}\left(\omega \mathbf{I}_{4}, \omega^{-1} \mathbf{I}_{4}\right), \\
& \gamma_{g_{2}, 9}=\operatorname{diag}\left(\omega \mathbf{I}_{2}, \mathbf{I}_{2}, \omega^{-1} \mathbf{I}_{2}, \mathbf{I}_{2}\right) .
\end{aligned}
$$

Here we have chosen $B_{12}=1 / 2, B_{34}=B_{56}=0$. The gauge group is $U(4) \otimes U(4)$. The matter consists of the following chiral superfields: $\Phi=(\mathbf{1 0}, \mathbf{1})(+2,0), Q=(\overline{\mathbf{4}}, \mathbf{4})(-1,+1)$, $\widetilde{Q}=(\overline{4}, \overline{\mathbf{4}})(-1,-1)$, where the $U(1)$ charges are given in parentheses. The tree-level superpotential, which can be derived using the standard techniques, reads:

$$
\mathcal{W}=\lambda \Phi Q \widetilde{Q}
$$

Note that this is precisely the second model discussed above except for the extra $U(1)$ 's. In fact, the first $U(1)$ is anomalous. It is broken by VEVs of some of the twisted closed

\footnotetext{
${ }^{7}$ Here we work with $2^{4-b / 2} \times 2^{4-b / 2}$ (rather than $2^{5-b / 2} \times 2^{5-b / 2}$ ) Chan-Paton matrices as we choose not to count the orientifold images of the corresponding D-branes.
} 
string sector chiral multiplets which transform non-linearly under the anomalous $U(1)$. The second $U(1)$ is anomaly free, and it is not difficult to see that it has no effect on the above discussion of VEV stabilization.

Note that in the above construction we have D9-branes. We can T-dualize to arrive at the corresponding Type I' vacuum with, say, D3-branes. Then the space transverse to D3-branes is $\mathcal{M}_{3}$. The point in the moduli space with the unbroken gauge group corresponds to the brane configuration where all D3-branes are sitting on top of the corresponding orientifold plane. The flat directions along which $\langle\Phi\rangle \neq 0,\langle\widetilde{Q}\rangle \neq 0$ and $\langle Q\rangle \neq 0$ then correspond to the branes moving off the orientifold plane in the $z_{1}, z_{2}$ and $z_{3}$ directions, respectively. Thus, if $\Phi \neq 0$ then motion in the $z_{2}, z_{3}$ directions is not allowed due to the corresponding F-flatness conditions implied by the superpotential (18). On the other hand, as we have discussed above, the VEV of $\Phi$ is stabilized via supersymmetry preserving non-perturbative dynamics on this branch of the classical moduli space, so that D3-branes are stabilized at a finite distance from the orientifold plane.

- $b=4$. We have 8 D9-branes. The orientifold projection is of the $S O$ type. The solution to the twisted tadpole cancellation conditions is given by [1] :

$$
\begin{aligned}
& \gamma_{g_{1}, 9}=\mathbf{I}_{4}, \\
& \gamma_{g_{2}, 9}=\operatorname{diag}\left(\omega \mathbf{I}_{2}, \omega^{-1} \mathbf{I}_{2}\right) .
\end{aligned}
$$

Here we have chosen $B_{12}=B_{34}=1 / 2, B_{56}=0$. The gauge group is $U(4)$. The matter consists of one chiral superfield $\Phi=\mathbf{6}(+2)$. Note that we have anomalous $U(1)$ in this model which is broken by VEVs of the corresponding twisted closed string sector chiral multiplets? Note that there are no renormalizable couplings in this case. This model is then the same as the first model we discussed above. Once we T-dualize so that we have D3- instead of D9-branes, the motion of D3-branes in the $z_{3}$ direction is described by the $\Phi$ VEV. Note that D3-branes are frozen in the $z_{1}, z_{2}$ directions as the corresponding moduli are absent. As we saw above, the VEV of $\Phi$ in this model is stabilized via supersymmetry breaking non-perturbative dynamics, so that D3-branes are stabilized at a finite distance from the orientifold plane.

Here we should point out that the above open string spectra correspond to perturbative (from the orientifold viewpoint) sectors. However, unlike in some other cases (examples of which were recently discussed in [14]), non-perturbative orientifold sector states in these models become heavy and decouple once we blow up the orbifold singularities (which is required to break anomalous $U(1)$ 's) along the lines of [15].

Before we finish this note, we would like to make a few comments. First, it would be interesting to see whether in models of this type log-like corrections to the Kähler potential could stabilize branes at distances large compared with the string length along the lines of [16]. Also, the above discussion assumed dilaton stabilization. In a more realistic setup dilaton would have to be stabilized via the standard "race-track" mechanism [17], or via

\footnotetext{
${ }^{8}$ Note that in this model the corrections to the Kähler potential due to the anomalous $U(1)$ breaking are important for the quantitative analysis of brane stabilization. They, however, do not affect the qualitative picture of brane stabilization.
} 
a quantum modification of the moduli space [18 (also see [19]), or else via some other mechanism. It would be interesting to understand these issues more explicitly in the brane world context (for a general discussion see [9]).

Finally, we should point out that brane stabilization mechanisms similar to those discussed above were considered in 20] in the non-compact context where gravity decouples from the gauge theory dynamics. Here we consider consistent compact tadpole-free orientifold models. Brane stabilization in the latter context was also discussed in [21], where branes were argued to be stabilized via an interplay between non-perturbatively generated superpotential and an anomalous $U(1)$. However, as we have mentioned above, anomalous $U(1)$ 's in the orientifold vacua are generically broken by VEVs of the corresponding twisted closed string sector chiral multiplets, which ameliorates the effect of the anomalous $U(1)$ 's leaving behind the usual non-perturbative runaway superpotential (as in the first model discussed in this note).

I would like to thank Gia Dvali and Gregory Gabadadze for discussions. This work was supported in part by the grant NSF PHY-96-02074, and the DOE 1994 OJI award. I would also like to thank Albert and Ribena $\mathrm{Yu}$ for financial support. 


\section{REFERENCES}

[1] Z. Kakushadze, Nucl. Phys. B535 (1998) 311.

[2] J. Polchinski, Phys. Rev. Lett. 75 (1995) 4724.

[3] E. Witten, Nucl. Phys. B471 (1996) 135.

[4] J. Lykken, Phys. Rev. D54 (1996) 3693.

[5] N. Arkani-Hamed, S. Dimopoulos and G. Dvali, Phys. Lett. B429 (1998) 263; Phys. Rev. D59 (1999) 0860004.

[6] K.R. Dienes, E. Dudas and T. Gherghetta, Phys. Lett. B436 (1998) 55; Nucl. Phys. B537 (1999) 47; hep-ph/9807522;

Z. Kakushadze, Nucl. Phys. B548 (1999) 205; hep-th/9812163;

Z. Kakushadze and T.R. Taylor, hep-th/9905137.

[7] Z. Kakushadze, Phys. Lett. B434 (1998) 269; Phys. Rev. D58 (1998) 101901;

Z. Kakushadze and S.-H.H. Tye, Phys. Rev. D58 (1998) 126001.

[8] I. Antoniadis, N. Arkani-Hamed, S. Dimopoulos and G. Dvali, Phys. Lett. B436 (1998) 257 ;

G. Shiu and S.-H.H. Tye, Phys. Rev. D58 (1998) 106007.

[9] Z. Kakushadze and S.-H.H. Tye, Nucl. Phys. B548 (1999) 180.

[10] V. Rubakov and M. Shaposhnikov, Phys. Lett. B125 (1983) 136;

A. Barnaveli and O. Kancheli, Sov. J. Nucl. Phys. 52 (1990) 576;

G. Dvali and M. Shifman, Nucl. Phys. B504 (1997) 127; Phys. Lett. B396 (1997) 64.

[11] I. Antoniadis, Phys. Lett. B246 (1990) 317.

[12] G. Dvali and Z. Kakushadze, Phys. Lett. B426 (1998) 78;

Z. Kakushadze, G. Shiu, S.-H.H. Tye and Y. Vtorov-Karevsky, Int. J. Mod. Phys. A13 (1998) 2551.

[13] M. Bianchi, G. Pradisi and A. Sagnotti, Nucl. Phys. B376 (1992) 365;

Z. Kakushadze, G. Shiu and S.-H.H. Tye, Phys. Rev. D58 (1998) 086001.

[14] Z. Kakushadze, hep-th/9904007; hep-th/9904211; hep-th/9905033.

[15] Z. Kakushadze, Nucl. Phys. B512 (1998) 221;

Z. Kakushadze and G. Shiu, Phys. Rev. D56 (1997) 3686; Nucl. Phys. B520 (1998) 75;

Z. Kakushadze, G. Shiu and S.-H.H. Tye, Nucl. Phys. B533 (1998) 25.

[16] G. Dvali, hep-ph/9905204.

[17] See, e.g.,

N.V. Krasnikov, Phys. Lett. B193 (1987) 37;

L. Dixon, V. Kaplunovsky, J. Louis and M. Peskin, SLAC-PUB-5229 (1990);

J.A. Casas, Z. Lalak, C. Muñoz and G.G. Ross, Nucl. Phys. B347 (1990) 243;

T.R. Taylor, Phys. Lett. B252 (1990) 59.

[18] G. Dvali and Z. Kakushadze, Phys. Lett. B417 (1998) 50.

[19] E. Dudas, Phys. Lett. B375 (1996) 189.

[20] J. de Boer, K. Hori, H. Ooguri and Y. Oz, Nucl. Phys. B522 (1998) 20.

[21] J. Lykken, E. Poppitz and S.P. Trivedi, Nucl. Phys. B543 (1999) 105. 